\title{
Written medical discharge communication from an acute stroke service: a project to improve content through development of a structured stroke- specific template
}

\author{
Petra Mäkelä, Chiara Haynes, Katherine Holt, Arindam Kar
}

Imperial College Healthcare NHS Trust

\begin{abstract}
Specific guidelines for the content of discharge summaries from acute stroke services do not currently exist. The aims of this project were to assess the strengths and weaknesses of stroke discharge communication from Imperial College Healthcare NHS Trust, to develop a structured template to guide completion, and to re-audit discharge communication following its implementation. The audit compared local performance against record standards from the Academy of Medical Royal Colleges (1), which was augmented by criteria generated from the British Association of Stroke Physicians (BASP) Stroke Service Standards (2). Discharge information was examined within the Trust's Electronic Discharge Communication (EDC) system to determine the recording of selected items for consecutively discharged patients from the hyperacute and acute stroke units. The audit was repeated following implementation of a newly developed stroke-specific discharge summary template. Fifty-one EDC summaries were examined at baseline (July 2012) and 30 summaries at re-audit (January 2013). The criteria which showed low adherence initially and which showed the most significant improvement following the introduction of the template were the guidance on blood pressure and lipids targets (increased from $2 \%$ and $0 \%$ respectively at baseline, to $93 \%$ post intervention), and the driving and flying advice (from $3 \%$ to $79 \%$ ). Documentation was also seen to improve for measures of physical and cognitive function, discharge arrangements, and follow up plans.
\end{abstract}

This audit cycle has demonstrated improvement in the consistency of content within written discharge communication following the introduction of a structured stroke-specific template adhering to combined criteria from identified standards.

\section{Problem}

Organised stroke unit care has been demonstrated to reduce morbidity and mortality (3), and the last decade has seen significant advances in many aspects of the management of stroke. However, the quality of written discharge communication from acute stroke services is variable. Specific, comprehensive guidelines for the required content of discharge summaries from stroke inpatient services do not currently exist. Differences in formal and informal training for the writing of discharge summaries are likely to contribute to the variation in output observed. Other factors influencing the quality of the discharge summary writing include: the amount of time available to complete the task and the competing demands, the quality of medical records available on which to base the summary, and the degree of multidisciplinary team and senior support available for the process (4). At the time of inception of this audit, the acute stroke service on which it was based (comprising a hyperacute stroke unit and two acute stroke units) did not utilise a uniformly adopted template to guide the composition of discharge summary information.

\section{Background}

Hospital discharge summaries act as the primary means of communication at the time of transfer from the responsible inpatient team to the subsequent provider of care. In the context of discharge from an acute stroke service, the receiving team may be based in a variety of settings. For example, a discharged patient could transfer to a local Acute Stroke Unit following repatriation, a Rehabilitation inpatient unit, an Early Supported Discharge team, Community Rehabilitation team, Primary Care, or medical services overseas.

High-quality discharge summaries are generally considered to be essential for facilitating patient safety and continuity during transitions between care settings, and reducing emergency readmissions $(5,6)$. In addition, discharge summaries provide a pivotal source of background reference information when a patient returns for outpatient follow-up review, and for rapid assessment during a subsequent acute presentation, which in this context may involve a decision on safety and appropriateness of thrombolysis. Assessment is facilitated by the Electronic Discharge Communication (EDC) system, whereby the summaries are available for viewing by all authorised parties across the Trust. Increasing use of electronic forms of patient documentation brings opportunities for enhanced standardisation, in which structure of content plays a key role (7).

\section{Baseline Measurement}

A baseline audit of written discharge communication from the Imperial College Healthcare NHS Trust hyperacute stroke unit (Charing Cross Hospital) and acute stroke units (Charing Cross Hospital and St Mary's Hospital) was conducted. Consecutively discharged patients were included to give a pragmatic 'snap-shot' 
target sample size of around 50 records.

Included patients were admitted to these wards under the care of the Stroke team only (i.e. discharge summaries for 'medical outliers' on these wards, with non-neurovascular reasons for admission, were excluded). The baseline sample was selected from July 2012 during a time interval when the authors of discharge summaries, the Stroke SHOs, were stable in their posts and familiar with the processes involved. The majority of the Stroke SHOs were nearing completion of their Stroke attachment at that time, and so issues resulting from inexperience with Stroke discharge summary completion were therefore minimised. A data collection tool was developed in Excel and was piloted on three case records. It was subsequently adjusted before final implementation, following a team discussion.

Results from the baseline audit are shown (alongside re-audit results) in Table 1.

See supplementary file: ds2210.pdf - "Table 1 - Adherence to Criteria for Stroke Discharge Summaries"

\section{Design}

In the absence of specific guidelines for the content of discharge summaries from acute stroke services, the audit compared local performance against general record standards extracted from the Academy of Medical Royal Colleges (1) and augmented this with critical information for aftercare providers for patients following stroke, as identified in BASP Stroke Service Standards (2).

The initial discharge summary template was developed. The template adhered to a combination of identified standards which had been used in the audit. Although not specified in these standards, additional headings of National Institutes of Health Stroke Scale score (NIHSS) on admission, and NIHSS postadmission (i.e. at 24 hours or pre-discharge) were also included. These were recommended for inclusion by senior stroke team clinicians upon consultation.

\section{Strategy}

PDSA Cycle 1: The initial discharge summary template, generated from the identified standards, was circulated among senior members and representative junior members of the acute stroke multidisciplinary team. Feedback on content was considered and modifications were incorporated. Additional items, which were highlighted as frequently absent in the team members' clinical experience of reading discharge summaries were also included through consensus decision making.

PDSA Cycle 2: The modified template was piloted by a sample of junior doctors across the acute stroke ward settings to assess the practicalities of its use in generating discharge communication in the real world context of competing demands and deadline pressures. The feedback was positive, in that the template assisted structuring of the discharge summary and prompted for inclusion of items which may have otherwise been omitted. Additional suggestions emerged from this pilot stage, since opportunities were identified to improve ease-of-use of the template. For example, the layout of the template was reformatted which enabled information to be more effectively transferred from the Word document into the Trust EDC system.

PDSA Cycle 3: The template was rolled out to all clinicians involved in producing discharge summaries. This involved incorporating the template into the standard practice of Stroke SHOs when writing the discharge summary and a review of the content by Stroke Registrars or Consultants. Feedback was sought following general implementation of the template. Several issues were identified regarding practicalities of the template lay-out and further amendments were incorporated. The updated template was then redistributed.

PDSA Cycle 4: A post-intervention audit was carried out. The intention of the repeat audit at this stage was to allow early identification of effectiveness of the template and guide further modification as necessary. Findings were disseminated through email summaries and by presentation at the departmental academic meeting, where further feedback and development points were discussed.

\section{Results}

The re-audit was carried out approximately three months after implementation of the structured template (January 2013). Consecutively discharged patients were included to give a pragmatic 'snap-shot' target sample size of 30 records for the reaudit. A slightly smaller sample size was chosen for this re-audit, since the aim was to assess the effects of the template soon after its implementation in order to guide further efforts.

Table 1 presents the results of the audit at approximately three months after implementation of the structured template, compared to baseline audit findings.

Several areas were found to have been completed to high levels (>90\% adherence) at both baseline and at re-audit: diagnosis, comorbidities, reason for admission, clinical narrative, physical examination, and brain imaging results.

In addition, the following areas were found to have been moderately well completed (60-89\%) both at baseline and re-audit: ECG findings, chest X-ray report, carotid Doppler findings (where applicable), glucose +/or lipids results, outstanding actions, whom responsible and a time-frame specified for outstanding actions. These areas were not significantly affected following implementation of the template.

However, areas which showed significant improvement, from low to moderate adherence at baseline to high adherence at re-audit, were: specified blood pressure and lipids targets, discharge arrangements and follow-up plan.

In addition, the following areas showed low adherence at baseline 
$(<60 \%)$, with improvement to moderate adherence at re-audit (60-89\%): NIHSS on admission, measures of physical function (Barthel/modified Rankin scale) on admission and discharge, measure of cognitive function, driving and flying advice and a plan for community, and specialist services input.

Areas of poor completion, found to have persistently low adherence at both baseline and re-audit ( $<60 \%)$, were: NIHSS post-admission, procedures as specified in a separate section from the clinical narrative, psychological assessment, and blood pressure on discharge.

Additional significant areas in which documentation was lacking both at baseline and re-audit were the patient's expressed wishes or concerns and relevant legal information (although documentation here improved from $5 \%$ at baseline to $47 \%$ at re-audit). However as this audit did not include a correlation of documentation within the discharge summaries to that recorded within the patients' full medical notes, it was not possible to identify those for whom this represented omission and those for whom the information was not required.

Structured wording was included within the introduced template regarding blood pressure and lipids targets (intended to assist in Primary Care reviews for secondary prevention post-discharge) and advice regarding driving and flying following a TIA or stroke (intended to reinforce verbally discussed patient guidance). Each of these areas showed significantly improved completion, from very low levels ( $0-3 \%$ across these areas) at baseline.

See supplementary file: ds2208.doc - "ICHT Stroke Discharge Summary Template"

\section{Lessons and Limitations}

Those areas found to have high levels of completion are likely to reflect those in which a robust system for documentation exists within the recorded source data. For example, there was an improved inclusion of a NIHSS score on admission following the introduction of the template specifically requesting this information, which should have been readily available within patients' medical notes (it is also prompted for within the Stroke Admission Clerking Proforma). However, despite a prompt for NIHSS score on discharge within the new template also, completion of this item remained low (at 53\%). This is likely to represent inconsistencies in documentation within the source data in patients' notes, as a standardised process for recording NIHSS scores pre-discharge had not been established at the time of the re-audit. A similar issue affects documentation of physical functioning (Bathel index and modified Rankin scale scores) pre-discharge.

Areas which require further assessment include the extent to which discharge information is reviewed with the patient, family and other caregivers. An emphasis should be placed upon on assessing the acceptability and their comprehension of discharge information.

In addition, further consideration could be given to the liaison of multidisciplinary teams when writing discharge summaries.
Measures could be taken towards formalising the team processes in order to allow ways to coordinate specialist information from different professionals into one document. Further items that would be useful to incorporate are likely to emerge as the template becomes embedded into practice, and evolution of the template will be required.

Ideally the template would be integrated into the Trust's Electronic Discharge Summary system. This would avoid the need for a second application, i.e. constructing the document in Word and then transferring into the EDC system.

Several significant areas were identified with persistently low completion rates in the re-audit. These warrant further attention in terms of broader strategies within clinical processes, i.e. before reaching the stage of discharge summary writing. These areas include psychological assessment, the patient's expressed wishes or concerns, and relevant legal information.

\section{Conclusion}

This project has demonstrated that the introduction of a structured template to the acute stroke service at the Imperial College Healthcare NHS Trust improved the consistency of completion of discharge summaries according to criteria extracted from the Academy of Medical Royal Colleges Guide to Record Standards and BASP Stroke Service Standards.

This is significant for a service in which junior members of the clinical team rotate frequently and may not have a high degree of personal familiarity with the patients for whom summaries are to be composed, and are often writing summaries in the context of time pressures, competing demands, and discharge deadlines. The template should be considered to be a tool that will require future modification according to practicalities of its use in the context of evolving electronic patient records systems, stroke service requirements, and relevant emerging guidelines.

\section{References}

1. Academy of Medical Royal Colleges A Clinician's Guide to Record Standards - Part 2. Standards for the structure and content of medical records and communications when patients are admitted to hospital; Section Four - Discharge Summary. 2008.

http://www.rcoa.ac.uk/sites/default/files/FPM-cliniciansguide2.pdf.

2. British Association of Stroke Physicians. Stroke Service Standards. 2010. http://www.basp.ac.uk/Portal/2/2010\%20BASP\%20Stroke\% 20Service\%20Standards.pdf.

3. Stroke Unit Trialists' Collaboration. Organised inpatient (stroke unit) care for stroke. Cochrane Database of Systematic Reviews 2007. 4:CD000197.

4. Greysen SR, Schiliro D, Horwitz LI, Curry L \& Bradley EH. "Out of sight, out of mind": Housestaff perceptions of qualitylimiting factors in discharge care at teaching hospitals. 


\section{BMJ Quality Improvement Reports}

Journal of Hospital Medicine. 2012; 7(5), 376-381.

5. Kripalani S, LeFevre F, Phillips CO. Deficits in

communication and information transfer between hospitalbased and primary care physicians: Implications for patient safety and continuity of care. JAMA. 2007;297: 831-841.

6. Coleman EA. Falling through the cracks: challenges and opportunities for improving transitional care for persons with continuous complex care needs. J Am Geriatr Soc. 2003;51:549-55.

7. Ferranti JM, Musser RC, Kawamoto K, Hammon E. The clinical document architecture and the continuity of care record: a critical analysis. J Am Med Inform Assoc. 2006;13: 245-52.

\section{Declaration of interests}

Nothing to declare.

\section{Acknowledgements}

Dr Diane Ames

Dr Thomas O'Brien 